\title{
High precision refraction measurements by solar imaging during occultation: results from SOFIE
}

\author{
Larry Gordley, ${ }^{1, \star}$ John Burton, ${ }^{1}$ Benjamin T. Marshall, ${ }^{1}$ Martin McHugh, ${ }^{1}$ \\ Lance Deaver, ${ }^{1}$ Joel Nelsen, ${ }^{2}$ James M. Russell, ${ }^{3}$ and Scott Bailey ${ }^{4}$ \\ 'GATS, Incorporated, 11864 Canon Boulevard, Suite 101, Newport News, Virginia 23606, USA \\ ${ }^{2}$ Space Dynamics Laboratory, Utah State University, 1695 North Research Park Way, \\ North Logan, Utah 84341, USA \\ ${ }^{3}$ Center for Atmospheric Sciences at Hampton University, 23 Tyler Street, Hampton, \\ Virginia 23668, USA \\ ${ }^{4}$ Virginia Polytechnic Institute and State University, Blacksburg, Virginia 24061, USA \\ *Corresponding author: I.I.gordley@gats-inc.com
}

Received 13 April 2009; revised 5 August 2009; accepted 7 August 2009; posted 11 August 2009 (Doc. ID 110001); published 20 August 2009

\begin{abstract}
A new method for measuring atmospheric refraction angles is presented, with in-orbit measurements demonstrating a precision of $\pm 0.02 \operatorname{arcsec}( \pm 0.1 \mu \mathrm{rad})$. Key advantages of the method are the following: (1) Simultaneous observation of two celestial points during occultation (i.e., top and bottom edges of the solar image) eliminates error from instrument attitude uncertainty. (2) The refraction angle is primarily a normalized difference measurement, causing only scale error, not absolute error. (3) A large number of detector pixels are used in the edge location by fitting to a known edge shape. The resulting refraction angle measurements allow temperature sounding up to the lower mesosphere. () 2009 Optical Society of America

OCIS codes: $\quad 120.5710,280.4788,280.4991,280.5715,040.6808$.
\end{abstract}

\section{Introduction}

Thermal sounding of planetary atmospheres using occultation (Fig. 1) is a well-developed methodology. The approach is to infer the density profile, then compute temperature $T$ and pressure $P$ using the barometric differential equation assuming hydrostatic equilibrium of an ideal gas:

$$
d P=-P g d z /(R T)=-\rho g d z
$$

The value $g$ is the gravitational constant, $\rho$ is density, $z$ is height, and $R$ is the universal gas constant.

Density is usually inferred by either measuring the transmission profile of a known absorber, such as $\mathrm{CO}_{2}$ or $\mathrm{O}_{2}$, or the refraction angle profile. Here

0003-6935/09/254814-12\$15.00/0

(C) 2009 Optical Society of America we present a new approach to refraction angle measurement, which simply requires the measure of the observed vertical angular extent of the solar image as a function of time.

Refraction measuring methods fall primarily into two categories: (1) observation of the bending of light from a celestial source (examples are stellar and solar occultation measurements) and (2) measurement of the Doppler shift of a transmitted signal as a function of time, as the transmitter is being occulted relative to the receiver. This allows path length bending and, therefore, the refraction angle to be inferred. Examples include transmitters on planetary probes with Earth or deep-space receivers.

Stellar occultation was suggested by Jones et al. [1] and demonstrated by Grechko et al. [2], White et $\overline{a l}$. [3] in the 80s, and more recently by Vervack et al. [4] and others. Ward and Herman [5] attempted to use data from the solar occultation Stratospheric Aerosol 
and Gas Experiment (SAGE) to measure refraction angles. Most found that pointing knowledge limited resulting temperature retrievals to the mid stratosphere and below.

The second method, radio occultation, has been used for sounding of planetary atmospheres, e.g., Kursinski et al., Ware et al., Hocke, Steiner et al., and Fjeldbo et al. [6-10], and is now being applied with great success using the GPS network and orbiting receivers. These measurements are providing a wealth of density, temperature, and pressure information up through the stratosphere and into the lower mesosphere.

\section{General Measurement Analysis}

The primary objective of this paper is to describe a new optical technique for accurate measurement of bending angles. The angles are obtained by measurement of the observed angular extent of the solar image. The bending angle $\alpha$ as a function of the observed impact angle $\theta^{O}$ (see Fig. 1) is inverted to temperature and pressure as a function of altitude using the same technique described in Ward and Herman [5]. Those details are not presented here, but to understand the measurements it is useful to outline the process, which requires a careful definition of the parameters in Fig. 1:

$r^{S}=$ Spacecraft radius defined as distance from center of curvature (COC) to spacecraft.

$r^{G}=$ Radius of point of closest approach (PCA) of a geometric line from spacecraft to point of origin on the Sun of observed ray.

$r^{O}=$ Radius of apparent PCA of observed ray. $r^{O}=r^{S} \sin \left(\theta^{O}\right)$.

$r^{T}=$ The true radius at perigee of the observed (refracted) ray.

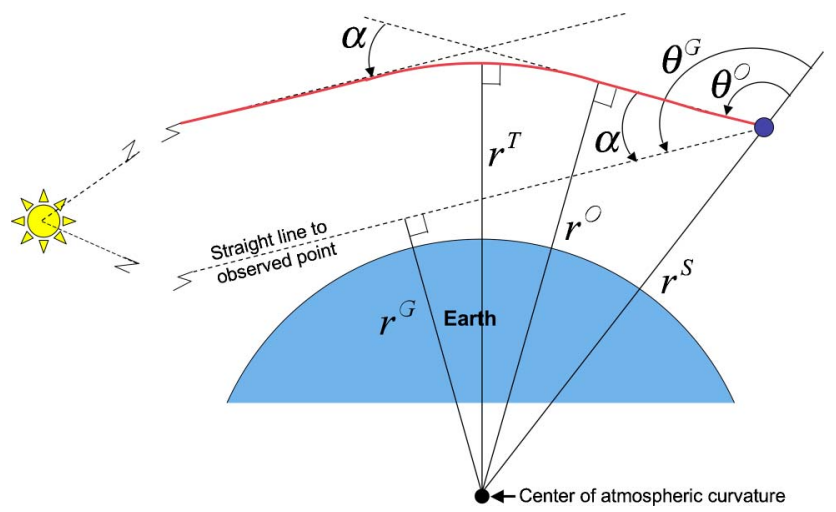

Fig. 1. (Color online) Refraction geometry. The red line depicts the path of light through the Earth's limb to the sensor, shown as a large solid dot. The refraction angle, $\alpha$, is the change in direction of the ray from when it enters the atmosphere to where it is received at the sensor. This is approximately the difference between the geometric angle and the observed impact angle at the satellite $\left(\theta^{G}\right.$ minus $\left.\theta^{O}\right)$. The radius at the impact tangent point, the point of closest approach for the observed ray at angle $\theta^{O}$, is called the impact radius, $r^{O}$. The altitude above the surface for the same point is called the impact altitude. The true refracted point of minimum perigee is labeled $r^{T}$.
$\theta^{O}=$ Angle of observed ray at the spacecraft (relative to vertical at the spacecraft).

$\theta^{G}=$ Angle at spacecraft of geometric line to Sun.

$\alpha=$ Refraction angle defined as $\theta^{G}-\theta^{O}$. To simplify the explanation we make the approximation of a spherically symmetric system where Earth, orbit, and density surfaces are concentric. In practice, the processing models explicitly address the differences from these assumptions. Note that the $\alpha$ at the top left of Fig. 1 is the true refraction angle, which is not exactly equal to the lower right $\alpha$ because the geometric line and refracted ray between the Earth and Sun are not perfectly parallel. However, this difference is always a negligible fraction of the refraction angle and is easily calculated.

Both the optical and radio methods obtain the refraction (bending) angle, $\alpha(t)$, as a function of time. From orbital mechanics $r^{S}, r^{G}$, and therefore $\theta^{G}$ are known as a function of time. Given the measured $\alpha$, and known $r^{S}, r^{G}$, and $\theta^{G}$ as a function of time, $r^{O}$ can be determined as a function of time, which provides the refraction angle $\alpha$ as a function of $r^{O}$.

This allows the index of refraction, $n$, to be calculated with an Abel transform of a function composed of refraction angle, $\alpha$, and impact radius, $r^{O}[\underline{5}, \underline{10}, \underline{11}]$ :

$$
n\left(r_{0}^{O}\right)=\exp \left[\frac{1}{\pi} \int_{r_{0} O}^{\infty} \frac{\alpha\left(r^{O}\right)}{\left[\left(r^{O}\right)^{2}-\left(r_{0}^{O}\right)^{2}\right]^{1 / 2}} \mathrm{~d} r^{O}\right] .
$$

Subscript 0 indicates the lowest level of the integration, which is defined by the ray appearing to pass through $r_{0}{ }^{O}$, but actually passing through $r_{0}{ }^{T}$. Every point (value of $r^{O}$ ) in the integration is consistent with an observed ray passing through a corresponding $r^{T}$. See Fig. 1 for a depiction of the various angles and radii.

Following convention, the Abel transform in Eq. (2) is written as a function of $r_{0}{ }^{O}$, the impact radius (unrefracted point of closest approach). However, the value of $n$ physically occurs at $r_{0}{ }^{T}$, the true point of closest approach (ray perigee). In fact, in the literature (e.g., [5]), the left side of Eq. (2) is sometimes written as $\bar{n}\left(r_{0}{ }^{T}\right)$ in an attempt to avoid confusion. For spherically symmetric atmospheres, $r^{T}$ is related to $r^{O}$ by

$$
r^{O}=r^{T} n\left(r^{T}\right)
$$

The atmospheric density $\rho$ can now be determined from $\rho=c(n-1)$, where $c=\rho_{\mathrm{STP}} /\left(n_{\mathrm{STP}}-1\right)$. Subscript STP indicates standard temperature and pressure $\left(0.0^{\circ} \mathrm{C}\right.$ and 760 Torr $)$. This work uses $n_{\mathrm{STP}}-1$ from Edlen [12]. Improvements over the years, for example, Ciddor and Hill [13], have not changed the accepted values of $n$ for dry air more than a few hundredths of a percent. (We issue a note of caution. The Edlen formula are for 760 Torr and $15^{\circ} \mathrm{C}$, not $0{ }^{\circ} \mathrm{C}$. This has also led to incidents of error that have propagated through the literature.) Using Eq. (1), the density profile is then integrated top down over 
altitude to determine pressure, $P$, as a function of altitude.

\section{Refraction Measurements by the SOFIE Instrument}

Traditionally, determining the refraction angle by observation of a celestial source from orbit has required an accurate knowledge of platform attitude. By eliminating the dependence on attitude information, the SOFIE instrument [14] has demonstrated refraction angle measurements nearly two orders of magnitude more accurate and precise than most previous stellar or solar occultation measurements.

The refraction angle as a function of impact radius $r^{O}$ can be obtained without pointing information by simply measuring the observed angular separation of two celestial points as they are being occulted by a planetary atmosphere. In this case the points are the top and bottom edges of the Sun, and the occultation is observed from an Earth orbiting satellite. This is equivalent to measuring the solar image angular extent $E(t)$ as a function of time. That extent is the observed angle subtended by the solar image in the elevation direction. Given the satellite ephemeris, a refraction angle profile as a function of the impact angle can be inferred, and the impact angle converted to the impact altitude. To our knowledge, this (now patented) approach of simultaneously measuring the angular separation of two points, has not been done before SOFIE.

Figure 2 illustrates the relative shapes of the solar image as it appears from the satellite. As the solar image sets through apparent tangent altitudes, the angular extent is measured by imaging the Sun on a 2D detector array and tracking both the top and bottom edge of the Sun simultaneously.

We adopt the following convention: Superscript's $G$ and $O$ indicate geometric and observed, respectively. Subscripts $T$ and $B$ indicate solar image top and bot-

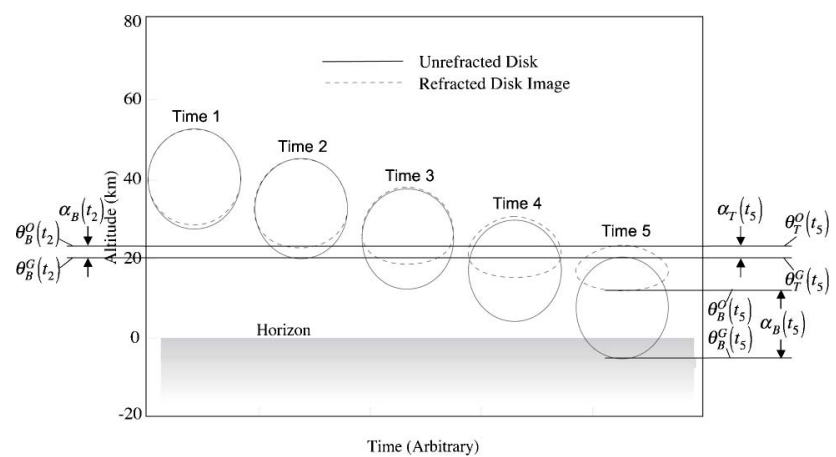

Fig. 2. (Color online) Refracted and unrefracted images of the setting Sun at five different snapshot times as they would appear from a low-Earth orbit. The chosen elapsed time between image 2 and image 5 is the time, $\Delta t$, required for the straight ray to the top edge of the Sun to move one unrefracted solar extent relative to the constant geopotential height horizon. $\Delta t=t_{5}-t_{2}$. Note that $\alpha_{T}\left(t_{5}\right)=\alpha_{B}\left(t_{5}-\Delta t\right)$. It can be shown that $\alpha_{B}(t)=$ $E_{o}-E(t)+\alpha_{B}(t-\Delta t)$, where $E_{o}$ is the unrefracted vertical extent and $E(t)$ is the refracted (observed) vertical extent (note $E_{o}=E(t)$ above the atmosphere). tom edges, respectively. Angles are measured from spacecraft local vertical.

From Figs. 1 and 2 , it is easy to see that the refraction angle a the bottom edge of the refracted image is

$$
\alpha_{B}=\theta_{B}^{G}-\theta_{B}^{O}
$$

and at the top edge is

$$
\alpha_{T}=\theta_{T}^{G}-\theta_{T}^{O}
$$

Therefore

$$
\alpha_{B}-\alpha_{T}=\theta_{B}^{G}-\theta_{T}^{G}-\left(\theta_{B}^{O}-\theta_{T}^{O}\right)=E_{o}-E,
$$

where $E$ is the measured solar extent. Subscript "o" indicates an unrefracted (geometric, above the atmosphere) measurement.

Now including notation for function of time $t$ :

$$
\alpha_{B}(t)=E_{o}-E(t)+\alpha_{T}(t) .
$$

$E_{o}$ and $E(t)$ are measured. If $\alpha_{T}(t)$ were known, Eq. (5) would produce the desired refraction angle as a function of time. Now note that if a time increment $\Delta t$ is known, where $t-\Delta t$ is the time when $r_{B}^{O}(t-\Delta t)=r_{T}^{O}(t)$, then $\alpha_{T}(t)=\alpha_{B}(t-\Delta t)$.

For an unrefracted solar image with angular extent $E_{o}$, the true (unrefracted) top and bottom edge positions, $\theta_{T}^{G}$ and $\theta_{B}^{G}$, are related by

$$
\theta_{B}^{G}(t)=\theta_{T}^{G}(t)+E_{o}=\theta_{T}^{G}(t+\Delta t(t)) .
$$

$\Delta t(t)$ is the time required for $\theta_{T}^{G}$ to change by $E_{o}$. This description specifically addresses a "sunset," but a sunrise analysis is identical if time is reversed.

True positions are only observable above the refractive atmosphere. Below (through the atmosphere), only the impact positions are observed along lines of sight that appear to pass through the impact altitude, $r^{O}$. However, relationships similar to Eq. (6) hold for the impact positions, which are observable:

$$
\theta_{B}^{O}(t)=\theta_{T}^{O}(t)+E(t),
$$

and the key point is

$$
\theta_{B}^{O}(t)=\theta_{T}^{O}(t)+E(t)=\theta_{T}^{O}(t+\Delta t(t)) .
$$

That is, the bottom edge appears at an angular distance of $E$ from the top edge and the observed top edge $\theta_{T}^{O}$ will change by $E$ in the same amount of time, $\Delta t$, as the unrefracted vector moves $E_{o}$.

$\Delta t$ can be determined from known spacecraft ephemeris and the known, or exoatmospheric measurement of, solar extent $E_{o}$. For circular orbits with solar vector in the orbital plane, $\Delta t$ is constant because under such conditions $\theta^{G}$ changes at a constant rate, which is the orbital angular velocity. In general, 
$\Delta t$ is a function of time and must be found by using exact orbital mechanics codes to calculate the func$\operatorname{tion} \theta_{T}^{G}(t)$, which is then used to determine how much time it takes for $\theta^{G}$ to change by $E_{o}$.

Now, Eq. (ㅁ) becomes

$$
\alpha_{B}(t)=E_{o}-E(t)+\alpha_{B}(t-\Delta t) .
$$

$\alpha_{B}(t-\Delta t)$ is typically less than $4 \%$ of $\alpha_{B}(t)$ and can be estimated at the highest altitudes with sufficient accuracy. However, after $\alpha_{B}$ measurements have progressed for $\Delta t$ (time for the Sun to sink $E_{o}$ from the top measurement), $\alpha_{B}(t-\Delta t)$ is known.

Given $\alpha_{B}(t)$ from measurements and $\theta_{B}^{G}(t)$ from orbital mechanics, $\theta_{B}^{O}(t)$ is known from Eq. (4a), producing the refraction angle as a function of the impact angle and, therefore, the impact radius as well. From Fig. $1, r^{O}=r^{S} \sin \left(\theta^{O}\right)$.

For highly elliptical orbits with planes more oblique to the solar vector, $\Delta t$ will be slightly different than the time the $\theta_{T}^{G}$ changes by $E_{o}$ due to the oblateness of the Earth combined with the changing ray tangent point location. These subtitles can be explicitly calculated to determine a profile of $\Delta t$ as a function of $t$. However, even if ignored, the estimate of $\Delta t$ will typically induce errors in the measured refraction angle of under $0.1 \%$. This is a fractional error proportional to $\alpha_{B}$ and, therefore, does not significantly limit the sensitivity of the measurement at any altitude.

In summary, the only measurement necessary to infer a refraction angle profile is angular extent of the solar image as a function of time. By imaging the Sun on a 2D high density, fast frame-rate, detector array, the top and bottom edges of the Sun's image can be located simultaneously, which completely removes platform attitude knowledge as an error source.

The Sun size and distance is well known, allowing the unrefracted image to be used to calibrate the angular spacing of the detector pixels. Because the measurement is a difference between the measured refracted and unrefracted images, the inferred refraction angle incurs only a scale error equivalent to the pixel spacing scale error. For example, if the pixel spacing is determined to $0.1 \%$ uncertainty (easy to achieve), the solar extent measurements will have the same fractional error. Therefore, measured refraction angles will incur the same $0.1 \%$ fractional error regardless of the size of the refraction angle, which does not affect the precision of the measurement.

The measured refraction angle accuracy is limited only by the accuracy to which the fractional change in image extent can be determined from the detector array. By incorporating 300 pixels into the edge detection measurement, sampled at $100 \mathrm{~Hz}$, with results averaged over $0.25 \mathrm{~s}$, precision enhancements of nearly a factor of 100 are obtained relative to an approach that simply interpolates between two pixels.
Basically, the approach fits an edge model to the detector array data.

The system implemented on the SOFIE instrument is demonstrating measurements with uncertainties in solar image shrinkage, $\left[E_{o}-E(t)\right]$, on the order of $\pm 0.02 \operatorname{arcsec}(0.1 \mu \mathrm{rad})$ with a system that is less than optimum. This is allowing single occultation temperature retrievals up to altitudes of 55 to $65 \mathrm{~km}$ with $2-5 \mathrm{~K}$ precision at the highest altitudes.

\section{Hardware Implementation}

The SOFIE instrument was launched onboard the Aeronomy of Ice in the Mesosphere (AIM) satellite on 25 April 2007 and started science observations on 14 May 2007. SOFIE combines a 16 channel broadband infrared radiometer with a $2 \mathrm{D}$ detector array used to image the Sun at $705 \mathrm{~nm}$. In addition to determining the location of the solar image relative to the radiometer field of view (FOV), the Sun sensor measures the vertical extent of the solar image and the intensity of the incident solar radiation. The high precision of these last two quantities allows the retrieval of temperature and pressure independent of the radiometer data. The following describes the sensor design and data processing used to produce the precision necessary for the $T / P$ retrievals.

The detector used for the SOFIE Sun sensor is a Fillfactory NV STAR1000 space qualified complementary metal-oxide-semiconductor (CMOS) detector array consisting of $1024 \times 102415 \mu \mathrm{m}$ pixels. This focal plane detector array (FPA) subtends $2^{\circ} \times 2^{\circ}$ of the far field. The resolution at the center of the detector is approximated by an Airy function of 17 arcsec from the center to the first dark ring. This resolution degrades by a few percent from the detector center to the edge. Pixel pitch was found to subtend $7.1^{\circ}$ arcsec vertically and 7.18 arcsec horizontally at the center of the array. Therefore, the solar image spans roughly 270 pixels in both elevation and azimuth.

The detector is controlled by a 16 bit $16 \mathrm{Mhz}$ space qualified UTMC UT69R000 microcontroller. The FPA and microcontroller reside on a Sun sensor circuit board (SSB) separate from the primary SOFIE command and data handling $(\mathrm{C} \& \mathrm{DH})$ board and communicates with the C\&DH board via an RS-422 data link. The two boards are synchronized by a $100 \mathrm{~Hz}$ heartbeat provided by the C\&DH board [15].

The Sun sensor was designed to serve three primary purposes: (1) control the FOV steering mirror (the launch configuration did not include a steering mirror, so this function is not performed); (2) determine the location of the solar image on the FPA; and (3) record intensity data at various locations on the solar image. To accomplish this, the FPA is subsampled into rectangular windows or regions of interest (ROIs) that designate the pixels to be read based on location on the FPA. The use of ROIs minimizes the computational, storage, and telemetry resources required. 


\section{Onboard Processing}

The SOFIE Sun sensor flight software is an interrupt-driven state machine responding to a $100 \mathrm{~Hz}$ heartbeat from the C\&DH board and consisting of three primary states: search, coarse track, and fine track. Each primary state is divided into five substates to allow execution of the code within the $0.01 \mathrm{~s}$ constraint of the $100 \mathrm{~Hz}$ interrupt loop.

\section{A. Solar Image Acquisition}

Prior to the beginning of a SOFIE occultation event, the spacecraft maneuvers to point the SOFIE FOV toward the center of the Sun, which places the solar image on the FPA. Upon initialization, the Sun sensor locates the solar image on the FPA using a 2D signal-weighted center of mass technique. This methodology is described below and depicted graphically in Figs. 3 and 4 .

The first step locates the horizontal and vertical projections of the image on the FPA. In the equation below, vector $H$ is the horizontal projection of the image obtained by the FPA. The individual element $H[i]$ is obtained by summing the values of the pixels in the $i$ th row. Similarly, vector $V$ is the vertical projection of the image obtained by the FPA, the sum of the values of the pixels in the jth column.

$$
V[j]=\sum_{i=M_{1}}^{M_{2}} \operatorname{FPA}[i, j] \quad H[i]=\sum_{j=N_{1}}^{N_{2}} \operatorname{FPA}[i, j]
$$

where FPA $[i, j]$ is the reading of pixel $i, j$. The summation starts and stops at various index values $M_{1}$, $M_{2}, N_{1}$, and $N_{2}$ using a different modulus (i.e., interleave factor) for each state 16,8 , and 1 for "search," "coarse," and "track," respectively.

The next step is to determine the 2D signalweighted center of mass $[h c, v c]$ on the FPA in FPA coordinates:

$$
v c=\frac{\sum_{j}^{N} j \times V[j]}{\sum_{j}^{N} V[j]}, \quad h c=\frac{\sum_{i}^{M} i \times H[i]}{\sum_{i}^{M} H[i]} .
$$

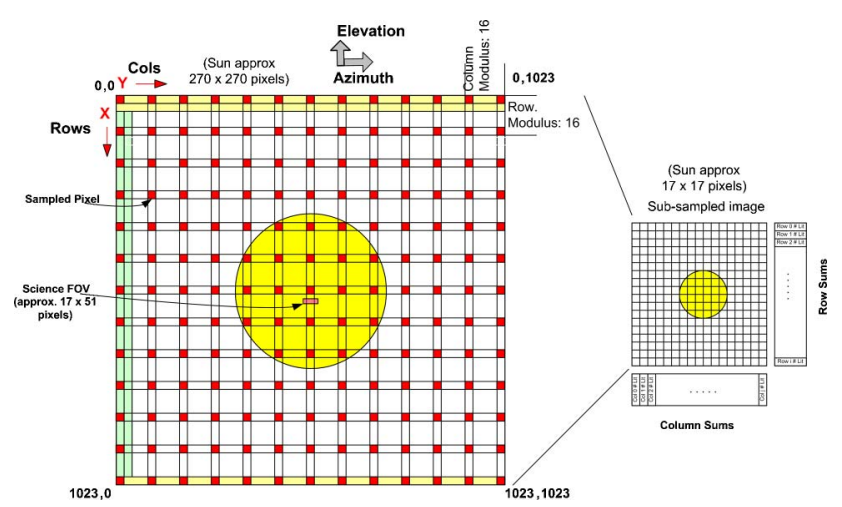

Fig. 3. (Color online) Search State (grid is not to scale). Search mode uses a modulus of 16 to allow full grid search in less than $0.01 \mathrm{~s}$.

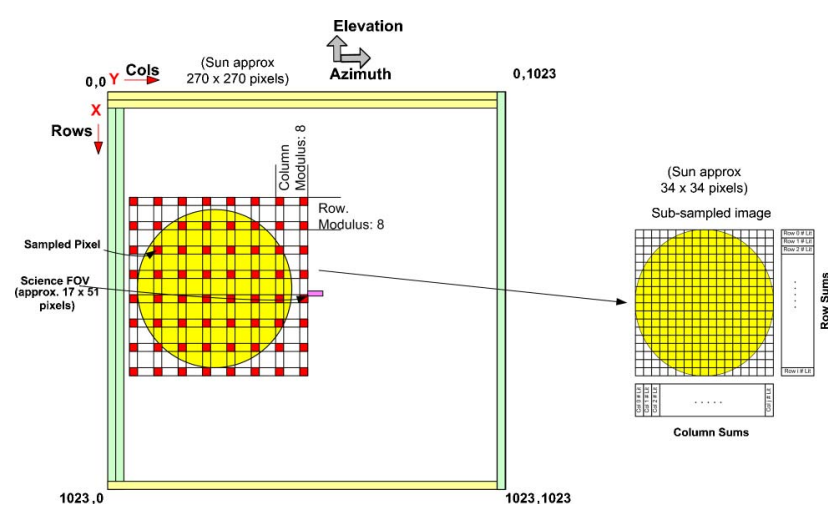

Fig. 4. (Color online) Coarse State. Position of the image is further refined after the Search State to provide location accuracy necessary for initiating Fine Track State.

Because of onboard computing constraints, the acquisition of the solar image is a two-step process consisting of the search and coarse tracking states. Both states use the center-of-mass algorithm described above, the primary difference being the ROI used to sample the FPA. "Search" mode locates the solar image on the FPA, and "coarse" track refines the location of the solar image with sufficient precision to start fine tracking in the "track" state.

After initialization, the Sun sensor enters the "search" state. As shown in Fig. 3, in search state the ROI is defined as the entire FPA subsampled by a factor of 16 in both dimensions, producing a $64 \times$ 64 image. The center-of-mass algorithm is then applied to this subsampled image to determine the location of the center of the solar image. This location is used to determine the initial ROI for coarse track.

As shown in Fig. 4, in coarse tracking the ROI is a $370 \times 370$ pixel area at the center of the solar image and subsampled by a factor of 8 in both directions, producing a $46 \times 46$ pixel image. The reduced subsampling interval provides a sufficiently precise location to transition into fine tracking. If the solar image is completely on the FPA, the locations of the two vertical (elevation) and two horizontal (azimuth) edges are determined. The four edge locations are found by starting at both ends of the row and column sum array and searching for values that bracket a predetermined threshold value. Interpolation between the bracket values is used to determine the row/column location of the edge to within the accuracy of the 10.5 format fixed point calculations (the 5 bits allocated to the fractional part of the fixed point number allow accuracy of approximately $1 / 32$ of a pixel). These four edge locations are then passed to the third primary state, fine track.

\section{B. Solar Image Tracking}

Fine track processing is the main operational state and serves two purposes: it provides a precise determination of the location on the FPA of the four solar edges (high and low elevation, high and low azimuth) and prepares the data to be transmitted to the ground station. The Sun sensor remains in fine track 
until the solar image is lost or it is commanded to reset. Figure 5 illustrates the processing that occurs in the fine track state. The edge locations determined by coarse track provide an initial estimate for the position of the four edge ROIs and the center ROI. The center ROI is a fixed offset from the top (high elevation) edge ROI. The fine track state is composed of 5 substates, each of which executes within the $0.01 \mathrm{~s}$ limit imposed by the $100 \mathrm{~Hz}$ interrupt. The processing of each ROI is the same: read-out the specified pixels from the FPA and sum the pixels along the rows (elevation edge and center ROIs) or columns (azimuth edge ROIs) to produce vectors of pixel sums. The edge sums are used to determine the location of the edge of the solar image. Onboard, this is accomplished by determining a value that is $25 \%$ of the difference between the minimum and maximum values within each sum vector. The row (or column) location of the $25 \%$ value within each of the vectors is determined using linear interpolation. ROI locations are updated after every fifth interrupt cycle $(20 \mathrm{~Hz})$.

\section{Telemetry}

Sun sensor data are placed in the telemetry buffer every $0.05 \mathrm{~s}(20 \mathrm{~Hz})$. The elevation edge ROIs are processed each $100 \mathrm{~Hz}$ cycle, averaged over five cycles, and placed in the telemetry buffer. The azimuth edge and center ROIs are processed every fifth $100 \mathrm{~Hz}$ cycle, and the resulting data are placed in the telemetry buffer. The onboard estimate of edge locations (also referred to as tracking values) are placed in the telemetry at the same time as the elevation ROI sums data. These edge locations also define the center of the ROI for the various sum values. Inherent in the onboard processing is a spatial averaging over pixels embodied by the sum, reducing the impact

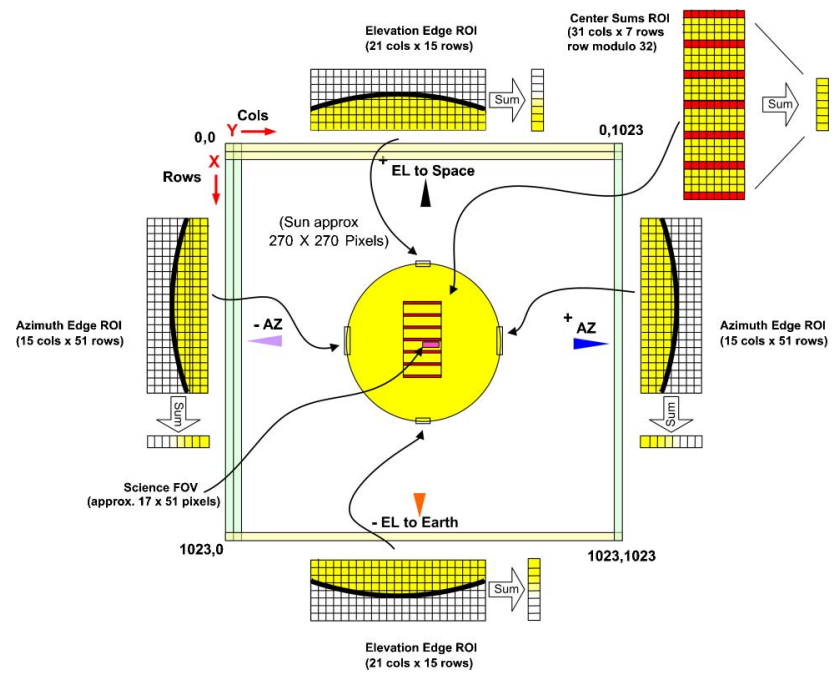

Fig. 5. (Color online) Fine Tracking State (illustrative-not to scale). The Sun image is kept within the FPA by the spacecraft. The science FOV is fixed relative to the Sun sensor FOV. Tracking the solar edges locates the science FOV relative to the solar image and provides the image extent used for refraction angle measurements. Center sums are used to measure atmospheric transmission, which is incorporated into the edge intensity model. of individual pixel noise, and a temporal averaging of the elevation edge data (five $100 \mathrm{~Hz}$ samples of the sums).

\section{Ground Processing}

\section{A. Tracking Timestamp Correction}

Time synchronization between the Sun sensor data and the radiometer science data is accomplished through the use of 16 bit free-running timers (FRT) on both the SSB and C\&DH boards. These FRTs have a resolution of $100 \mu \mathrm{s}$ and roll over every $6.5535 \mathrm{~s}$. The timestamps attached to the science data are generated by the FRT on the C\&DH board. The timestamps attached to the Sun sensor data are generated by the FRT on the Sun sensor board. Although the two hardware timers are identical and are synchronized at the start of an event, the timer on the SSB drifts by approximately $3.5 \times$ $10^{-3} \mathrm{~s} / \mathrm{s}$ relative to the C\&DH timer. If left uncorrected, this can accumulate to $0.5 \mathrm{~s}$ over a $150 \mathrm{~s}$ event, producing significant pointing registration error between Sun sensor and radiometer data. The C\&DH timer is used as the reference time and the Sun sensor timer is corrected to agree with it.

\section{B. Pixel Flat Field Correction}

Typical of most imaging sensors, the SOFIE Sun sensor exhibits a nonuniform pixel response to a uniform illumination source. This nonuniformity is corrected by the standard image processing technique of pixel flat-fielding. The calibration coefficients were obtained in the laboratory using an integrating sphere to provide uniform illumination across the Sun sensor FPA. A coefficient of relative response, $R[i, j]$, for each pixel was obtained using the equation below:

$$
R[i, j]=\frac{\operatorname{FPA}[i, j]}{\left(\frac{\sum_{i=1}^{M} \sum_{j=1}^{N} \operatorname{FPA}[i, j]}{M \times N}\right)},
$$

where $N=M=1024$ for the SOFIE FPA. Note that $\sum_{i=1}^{M} \sum_{j=1}^{N} R[i, j]=M \times N$. Therefore, the average relative response is unity. Dead pixels are handled as a special case and are indicated by having a relative response of zero.

Unlike a typical imaging application, which records individual pixel values, the SOFIE Sun sensor records row or column vector sums for each of the fine track ROIs along with the locations of those ROIs. The vector sums must be corrected for variations in pixel response.

For example, the vector measurement is

$$
H(i)=C \sum_{j=n_{1}}^{n_{2}} I(i, j) R(i, j)=\sum_{j=n_{1}}^{n_{2}} \operatorname{FPA}(i, j),
$$

where $I(i, j)$ is the irradiance on pixel $(i, j), C$ is a constant in units of (counts/irradiance), and $n_{1}$ and $n_{2}$ 
are the summation beginning and end points for the vector that is $\left(n_{2}-n_{1}+1\right)$ pixels in length.

We want to find a correction factor, $K$, to $H(i)$ that creates the value $H^{C}(i)$, which would have been measured had all pixel responses been a constant 1.0:

$$
H^{C}(i)=C \sum_{j=n_{1}}^{n_{2}} I(i, j)=K H(i),
$$

where $K$ is the correction factor, which is given by

$$
K=\sum_{j=n_{1}}^{n_{2}} I(i, j) /\left(\sum_{j=n_{1}}^{n_{2}} I(i, j) R(i, j)\right) .
$$

To use the above equation, it only requires a functional shape of $I$ over the pixels in the summation. Any error in the constant scale factor of $I$ will be removed by the ratio. Although the correction is applied, it was found to have little effect on results. This is because the edge was empirically modeled from a statistical determination of the shape.

\section{C. $5 \mathrm{~Hz}$ Readout Noise Removal}

The Sun sensor electronics were found to exhibit an unexplained $5 \mathrm{~Hz}$ noise. Periodically it introduces an additive $1 \%$ bias to the signal. During an affected readout operation, all the pixel values are increased by approximately $1 \%$, which in turn causes the sums data determined from those pixel values to be increased by $1 \%$. The periodic nature of this bias is such that it appears as a $1 \%$ increase added to the values for every fourth $20 \mathrm{~Hz}$ cycle, hence a $5 \mathrm{~Hz}$ noise. The black line in Fig. 6 shows this noise signature for one of the sums of the Low $X$ (high elevation) ROI.

The method used to remove this $5 \mathrm{~Hz}$ noise is based on the assumption that the $5 \mathrm{~Hz}$ noise is an additive bias and the baseline value is approximately the mean of the two adjacent samples. The sum values are sorted into a 2D array of constant FPA rows (or columns) versus time. The filter then uses a small window to detect the noise signature by obtaining

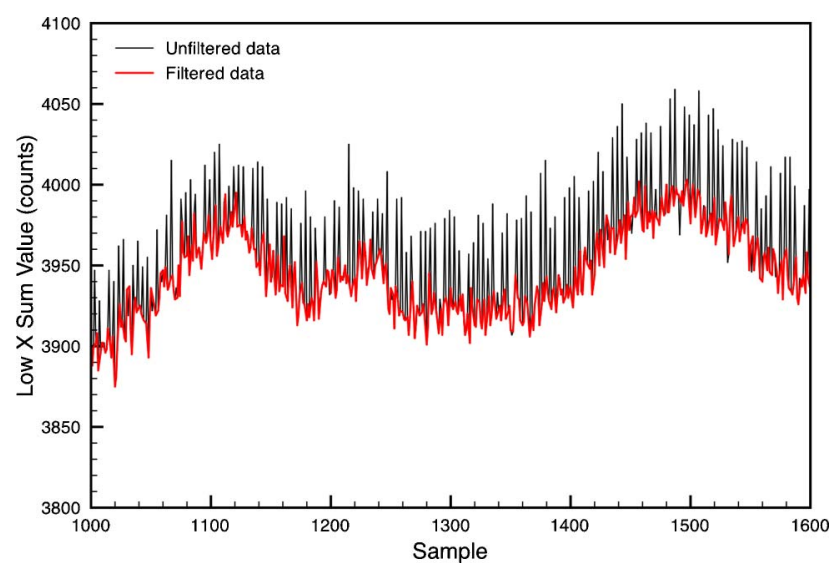

Fig. 6. (Color online) Removal of a unique readout noise produced by the Sun sensor electronics. four subsets of the row/column data. Each subset is the original data subsampled by a factor of 4 , each having a different sampling phase. The mean and standard deviation is then determined for each subset. If the mean of one subset differs significantly from the mean of the other subsets (the level of significance is determined by the standard deviation), then that subset is considered to have a $5 \mathrm{~Hz}$ noise signature. Filtering is accomplished by replacing affected samples with values from interpolation between adjacent samples. The red line in Fig. $\underline{6}$ shows the results of this filtering.

\section{Edge Model}

Accurate determination of the location of the top and bottom (elevation) edges of the solar image on the FPA is key to a precise determination of the vertical solar extent. Early theoretical analysis of the Sun sensor demonstrated a roughly $+/-0.25$ arcsec elevation uncertainty in the measurement using a linear interpolation method to find a specific intensity, similar to the previously described onboard method for determining the location of the solar edge. It was also noted that the elevation errors varied in a periodic fashion as the edge moved in a vertical direction. The period of the elevation error variation coincides with the pixel spacing of $\sim 7$ arcsec. This common shift-variant sampling artifact, frequently referred to as sample-scene phase error, is well understood $[16,17]$ and must be accommodated to facilitate improved precision in the solar extent measurement. It is primarily a result of the linear interpolation method for determining the edge.

The disadvantage of the linear intensity location method is that only two data points are used per edge, and it treats the top and bottom edges independently. Explicitly modeling the edge signal, including optical focus, sampling, and vector sums, then fitting the model to the measured vector sums reduces systematic errors and improves precision.

A high-resolution empirical model for the top and bottom edges was developed based on the analysis of 400 exo-atmospheric edge data sets (of 14 data points each-seven data points for the top edge and seven data points for the bottom edge) for 200 events spread over the first year of the mission. The edge data was normalized in both intensity and location, and was found to fit a Boltzmann function for both the top and bottom edges, with a fit quality $\left(R^{2}\right)$ value of greater than 0.9997 for both the top and bottom edges. The model is given in the equation below and displayed in Figs. 7 and 8. Note that the red dots represent the actual data and the black line is the Boltzmann model function. This exo-atmospheric edge model is then used to fit the edge data, the edge sums vectors.

$$
y=a 1+\frac{(a 0-a 1)}{\left(1+e^{\left(\frac{x-a 2}{a 3}\right)}\right)},
$$




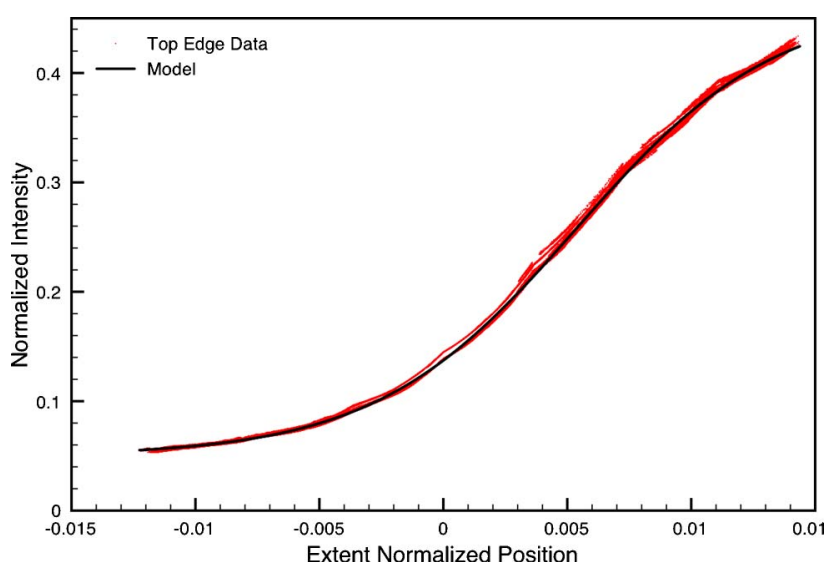

Fig. 7. (Color online) Empirical Top Edge Model. The black line is the Boltzmann function model for top edge. The red are the 560,000 individual data points from the top edge and the many events used to determine the parameters for the Boltzmann function.

where $x$ is the extent normalized location relative to the top edge, $y$ is the normalized intensity, and

$\begin{array}{ll}\begin{array}{c}\text { For the high elevation } \\ \text { (top) edge : }\end{array} & \begin{array}{c}\text { For the low elevation } \\ \text { (bottom) edge : }\end{array} \\ a 0=0.0500416 & a 0=0.595815 \\ a 1=0.464446 & a 1=-0.000650605 \\ a 2=0.00534758 & a 2=0.991427 \\ a 3=0.00404353 & a 3=0.00716971\end{array}$

During a typical occultation event, edge data is recorded at $20 \mathrm{~Hz}$, producing approximately 2500 sets of edge data, each consisting of 14 elevation edge data points (i.e., seven top edge data points and seven bottom edge data points) and 10 azimuth data points. During processing of an event, the exoatmospheric edge model is fit to each set of 14 elevation edge data points (total of top and bottom) using a Levenberg-Marquardt minimization algorithm. The minimization process has four adjustable (floating)

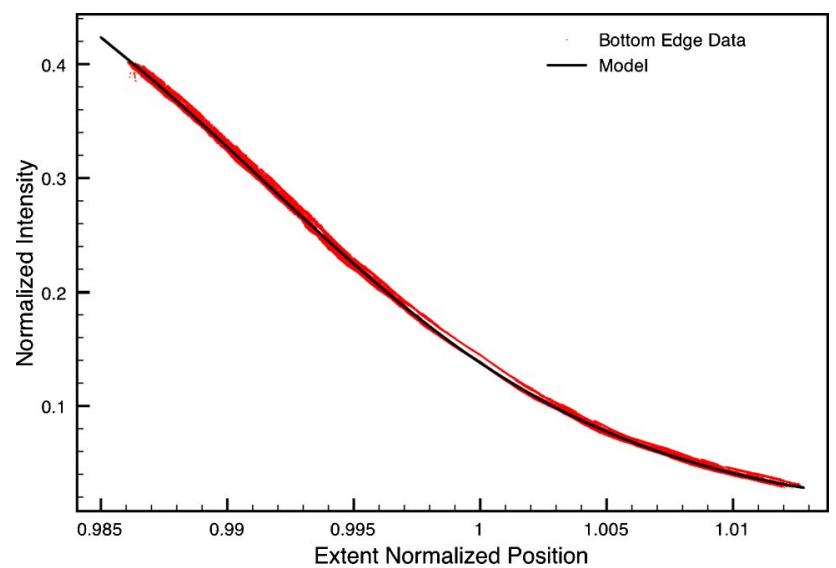

Fig. 8. (Color online) Empirical Bottom Edge Model. The black line is the Boltzmann function model for bottom edge. The red are the 560,000 individual data points from the bottom edge and the many events used to determine the parameters for the Boltzmann function. parameters, the location of the top edge, the distance between the top and bottom edge (solar extent), and the degree of attenuation of the model data for the top and the bottom edges. The scale of the extent is adjusted to be consistent with known solar extent for the relevant time of year. It was found that explicitly including edge contraction due to refraction in the edge model was unnecessary. The vertical shape of the edge model is dominated by the optical resolution, rendering error from refraction distortion of the image negligible. These parameters are iteratively adjusted until a good fit (typically $R^{2}>0.99$ ) is achieved. The use of this model-based approach provides us with solar extent precision in the 0.02 arcsec range.

\section{E. Coordinate Transformation}

The processing now switches from units of pixels to azimuth/elevation $(A Z / E L)$ in units of arcminutes. The $A Z / E L$ coordinate system is Earth relative so that $+E L$ is away from Earth. In this coordinate system, the top center point (TCP) is the edge of the solar disk furthest away from the Earth. The conversion from $X / Y$ (FPA) coordinates to $A / E(A Z / E L)$ coordinates is approximately

$$
\begin{aligned}
& E=\frac{(512-X) \times 7.10}{60}(\text { elevation in arcmins }), \\
& A=\frac{(Y-511) \times 7.18}{60}(\text { azimuth in arcmins }) .
\end{aligned}
$$

In addition to this transformation, a mapping function is used to account for apparent nonuniform pixel spacing caused by the optical angular distortion of the FOV on the FPA. This is a small effect that if ignored would result in a small fractional error (scale error).

The remaining processing to obtain the solar extent is trivial, determining the distance between the top and bottom solar edges and applying a 5-point $(0.25 \mathrm{~s})$ smoothing to the resulting solar extent data. The results are shown in Fig. 9, with the standard deviation plot determined by a $1 \mathrm{~s}$ (20 point) running standard deviation of the solar extent measurement. It is clear that the precision demonstrated in the plot significantly exceeds the 0.1 arcsec predicted in Gordley et al. [18]. Figures 10 and 11 show the measured refraction angle profile and the retrieved temperature profile, respectively, for the same event. The temperature profile is compared to the temperature profile retrieved from the SOFIE $4.3 \mu \mathrm{m}$ radiometer measurement.

\section{F. Temperature Retrieval and Results}

Figure 12 shows temperature profiles over Europe in the late fall of 2008 and summer of 2008 for two individual spacecraft sunrise events derived from SOFIE refraction angle measurements compared to National Centers for Environmental Prediction (NCEP) analyses extended with the 


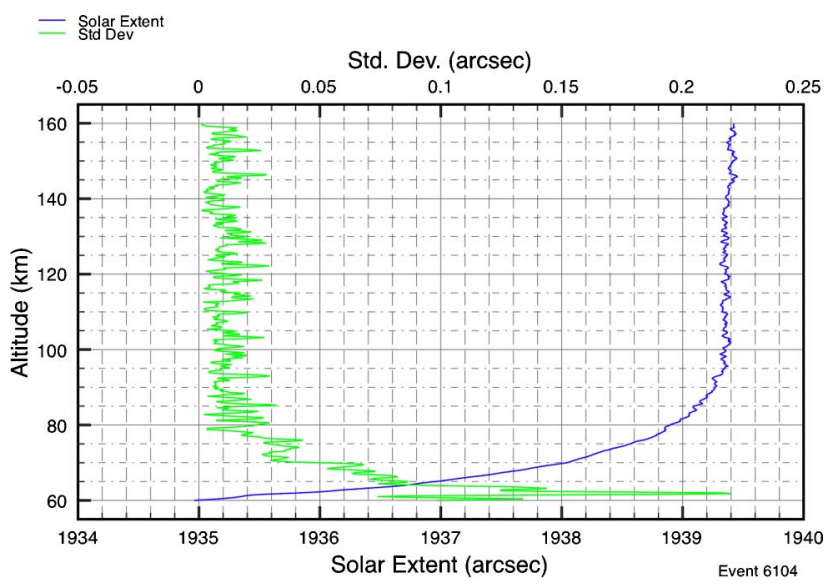

Fig. 9. (Color online) Solar Extent and 5-point running standard deviation for event 6104 . The green line is standard deviation of measured extent and blue is the measured extent.

Mass-Spectrometer Incoherent-Scatter (MSIS) data [19] simply for comparison. The black is the independent temperature and pressure profile (above $30 \mathrm{~km}$ ) derived from the $4.3 \mu \mathrm{m}$ channel. The excellent agreement near $60 \mathrm{~km}$ is achieved by using the temperature and pressure profile derived from the SOFIE $\mathrm{CO}_{2}$ channel for the upper boundary (above $60 \mathrm{~km}$ ). An analysis and validation of results will be presented in a future paper describing details of the retrieval approach. A brief outline of the retrieval is presented here.

The objective is to produce the best possible SOFIE temperature product, which is a combination of information retrieved using the $4.3 \mu \mathrm{m} \mathrm{CO}$ transmission measurements and the refraction angle measurement. First a temperature and pressure $(T / P)$ profile is retrieved using the $4.3 \mu \mathrm{m}$ channel and an assumed $\mathrm{CO}_{2}$ mixing ratio profile above $30 \mathrm{~km}$, which is extended below $30 \mathrm{~km}$ with the NCEP product,

Refraction Plot for Event 6104

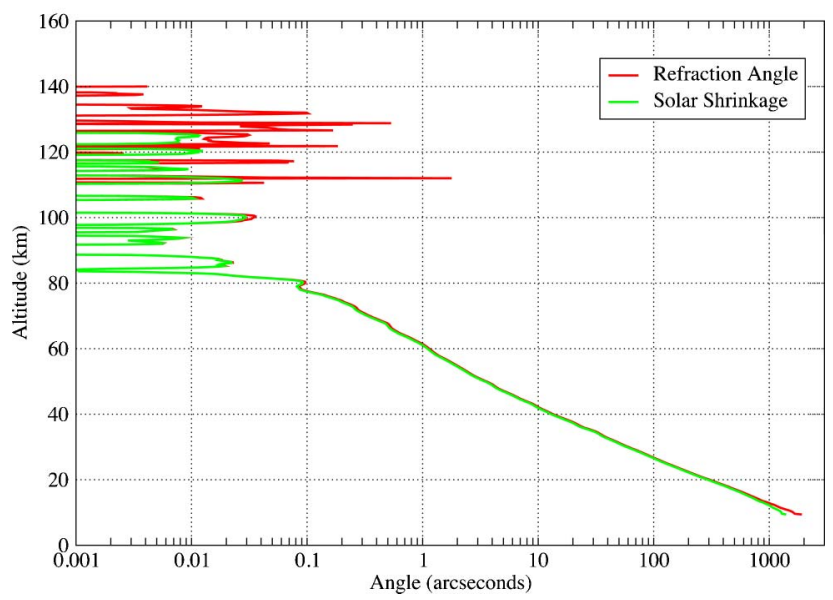

Fig. 10. (Color online) Measured "Solar Image Shrinkage" plotted with inferred refraction angle. "Shrinkage" is typically $4 \%$ (at high altitudes) less than the refraction angle at the bottom of the solar image.

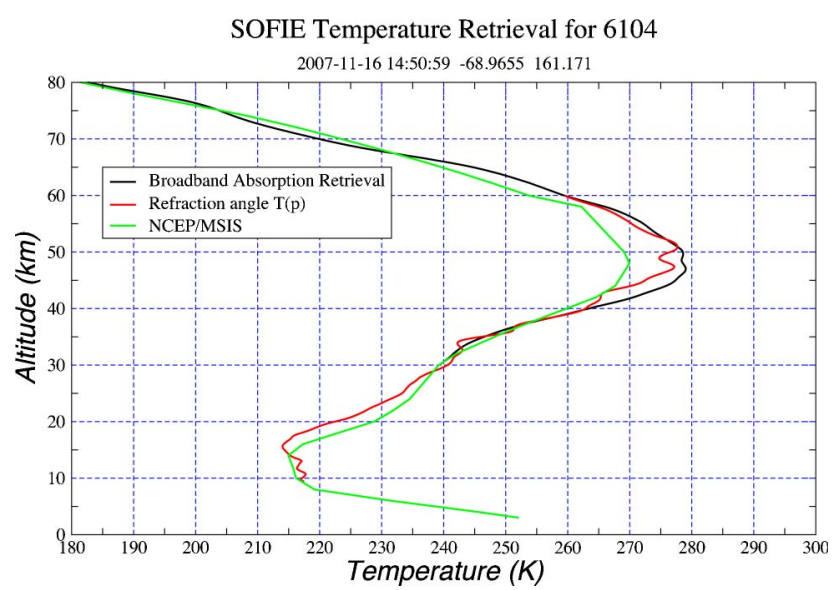

Fig. 11. (Color online) Temperature profile, retrieved from refraction profile in Fig. 10, compared to the NCEP/MSIS combined profile and profile retrieved from the SOFIE $4.3 \mu \mathrm{m}$ channel.

with a weighted merge applied between 30 and $40 \mathrm{~km}$.

Next, the $4.3 \mu \mathrm{m} T / P$ profile is used to simulate a refraction angle profile that is merged with the measured refraction angle profile. The merge window is
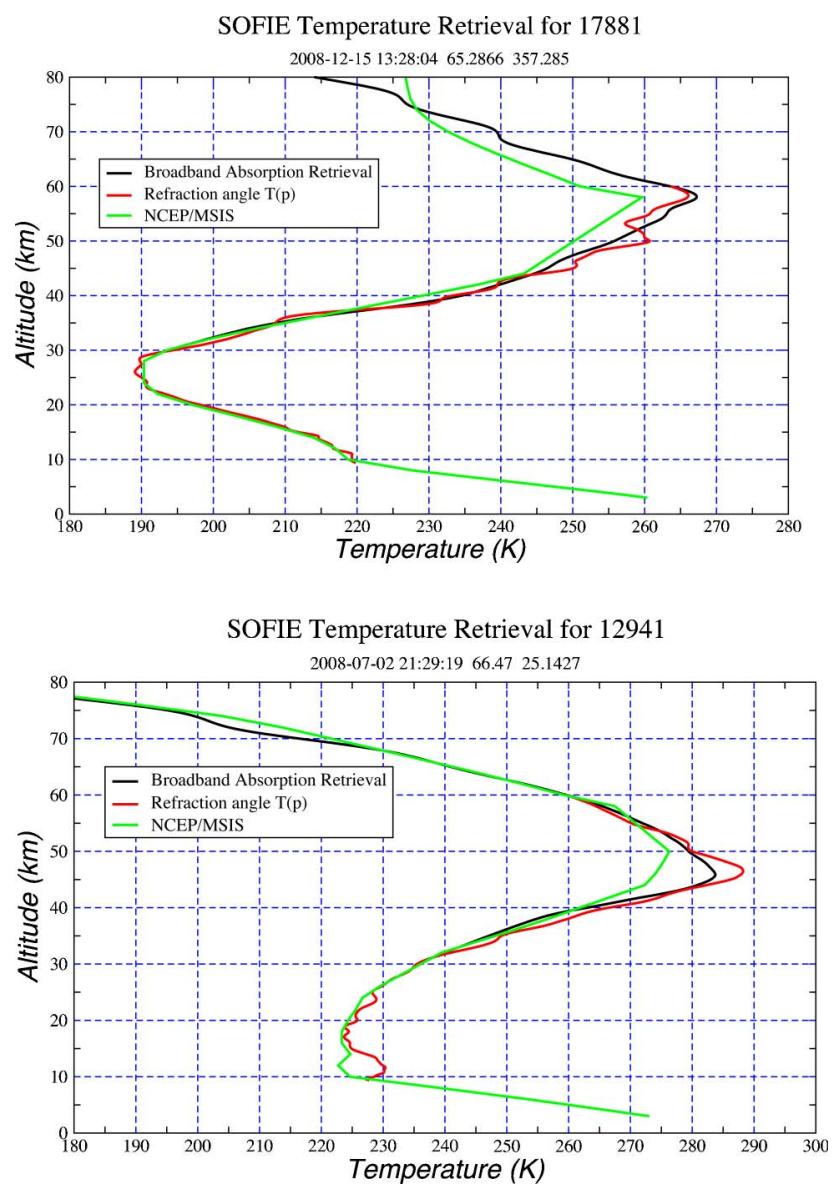

Fig. 12. (Color online) Comparison of temperature profiles for two events, late fall and summer of 2008. Black is the temperature retrieval from the SOFIE $4.3 \mu \mathrm{m}$ radiometer channel. The red is from the refraction angle analysis. The green, added for comparison, is from the NCEP product, extended above $50 \mathrm{~km}$ with MSIS. 
between altitudes that coincide with where the simulated 3.0 and 0.3 arcsec refraction angles occur. The merging process includes applying a constant refraction angle offset to the measured refraction angle profile. The offset is determined by minimizing the difference between the measured and the modeled refraction angles over the merge window, which typically occurs between 55 and $72 \mathrm{~km}$.

This offset approach was used because the dominant systematic error in the refraction angle measurement is the correction for drift in the $E_{o}$ measurement. Because this drift is rarely observed to be more than $0.05 \operatorname{arcsec}$ per $10 \mathrm{~km}$ of sunset $(\sim 3 \mathrm{~s})$, it rapidly becomes a negligible effect below the 3.0 arcsec level. The approach was found to nearly eliminate large faulty temperature variations at the top of the refraction retrieval $(>55 \mathrm{~km})$. Sources of the drift are not fully understood, however, they are likely related to shift-variant and thermal effects. For example, thermal expansion coefficients of the detector can induce a 0.015 arcsec change in measured solar extent per degree Celsius.

Because the SOFIE $4.3 \mu \mathrm{m}$ temperature product is believed to be most accurate near $60 \mathrm{~km}$, with density error typically at the $1 \%$ level or less, it produces an excellent upper boundary for the refraction angle retrieval. The merge uses a weighted average of the simulated and measured refraction angle profiles, linearly changing the weight through the window from all simulated above the 0.3 arcsec level to all measured below the 3.0 arcsec level. Therefore, the refraction profile used for a $T / P$ retrieval is purely a refraction angle measurement below the 3.0 arcsec level and is perfectly consistent with the $4.3 \mu \mathrm{m} \mathrm{T/P}$ results above the 0.3 arcsec level.

If the retrievals were to start with greater error in the upper boundary, the refraction temperature uncertainties increase with the errors exactly like those characterized by several other authors $[\underline{4}, \underline{5}]$. So we will not repeat such studies here.

The retrieval then uses the combined simulated and measured refraction angle profile to retrieve the index of refraction from Eq. (2) and temperature pressure from Eq. (1). The top of the profile gives the same $T / P$ result as from the $4.3 \mu \mathrm{m} \mathrm{CO}_{2}$ channel, gradually transitioning to purely refraction measurement results as the hydrostatic integration process proceeds downward through the merge window. Figure 13 shows a statistical comparison of refraction-based temperature profiles and $\mathrm{CO}_{2}$ transmission-based profiles. Mean profiles and SD about the mean difference are shown for three tests where the upper boundary was moved 0,5 , and $10 \mathrm{~km}$ higher than the standard described above. As can be seen, this does not significantly change the results below $60 \mathrm{~km}$. However, the SD increases with altitude above $60 \mathrm{~km}$ for the higher boundary cutoff. Note that the SD decreases toward zero, as expected, with altitude in the merge window as the refraction angle profile is weighted toward the simulated. When the SD becomes larger than $3-4^{\circ} \mathrm{K}$, the refrac-
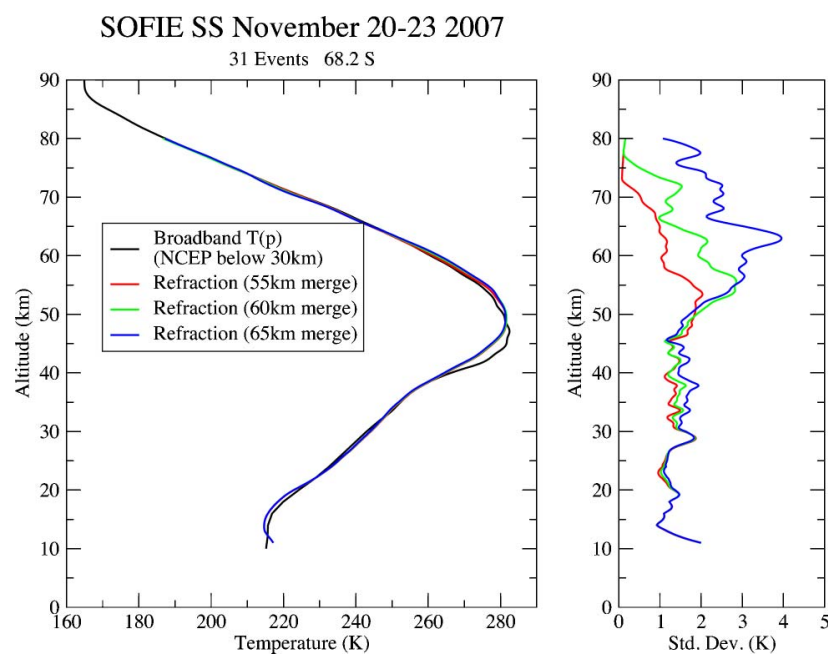

Fig. 13. (Color online) Statistical comparison of 31 temperature profiles retrieved from refraction angle versus those retrieved from $\mathrm{CO}_{2}$ transmission. Temperature and pressure from $\mathrm{CO}_{2}$ transmission is used to simulate a refraction angle upper boundary. The three comparisons are for three different upper boundary merge windows, which are roughly $15 \mathrm{~km}$ high with bottom starting at approximately 55,60 , and $65 \mathrm{~km}$. Also plotted is the standard deviation about the mean difference. (The bias between 40 and $50 \mathrm{~km}$ is a known error in the SOFIE temperature retrieval using the $4.3 \mu \mathrm{m} \mathrm{CO} \mathrm{CO}_{2}$ transmission and will be corrected in a future data release.)

tion temperatures are no longer considered better than the temperatures from the $\mathrm{CO}_{2}$ transmission. In fact, a small percentage of the more noisy refraction retrievals start to fail when the upper boundary is pushed above $60 \mathrm{~km}$.

The refraction retrievals are processed to achieve a vertical resolution of approximately $1 \mathrm{~km}$. This resolution is more than twice the $2.0 \mathrm{~km}$ resolution of products derived from the science data, which is quite evident in Fig. 12.

The SOFIE science data is sampled at $20 \mathrm{~Hz}$ with a $10 \mathrm{~Hz}$ bandwidth. The instantaneous science FOV at the $83 \mathrm{~km}$ tangent height is about $1.5 \mathrm{~km}$. The combination of spatial and temporal smoothing (the tangent point moves at roughly $3 \mathrm{~km} / \mathrm{s}$ ) produces an effective FOV of about $2.0 \mathrm{~km}$. The temperature retrieved from the $4.3 \mu \mathrm{m}$ transmission is composed of 10 independent interleaved retrievals, each having a $2 \mathrm{~km}$ grid point spacing with results interpolated to $0.2 \mathrm{~km}$. The ten profiles are then averaged to produce one profile.

The Sun sensor extent measurement has an effective instantaneous resolution of a few arcseconds (less than $0.1 \mathrm{~km}$ in the middle atmosphere). It is sampled at $100 \mathrm{~Hz}$, averaged and output at $20 \mathrm{~Hz}$ then averaged with a 5 point smoothing $(\sim 0.75 \mathrm{~km})$, and further refined by the edge-fitting algorithm before use in the retrieval algorithm. The final temperature product has an effective resolution of about $1 \mathrm{~km}$ and is interpolated to a $0.2 \mathrm{~km}$ grid coinciding with the science data. The potential for higher resolution at lower altitudes will be investigated; however, the breakdown of the spherical symmetry 
assumption for the limb geometry is a limiting factor, and has not been quantified.

\section{G. Validation of Precision}

A simulation study was performed to determine a theoretical limit to the precision of the solar extent measurement. The simulation model was based on an idealized Sun sensor processing train for the top and bottom elevation edge ROIs with the following assumptions: (1) The only noise present is pixel readout noise, which was estimated as 2 bits (4 counts out of 1024) and modeled as additive, zero-mean uniformly distributed random noise. (2) The location of the top and bottom edges were fixed providing a known solar extent. (3) The nonoise base values for each pixel are calculated using the edge model Boltzmann functions described in Subsection 6.D. Each time a pixel read is simulated, a random noise value is added to the base value. The average pixel values produced by a simulation of the entire sampling and onboard processing were used as input for the Levenberg-Marquardt minimization routine mentioned above to determine the location of the solar elevation edges, which gives us the solar extent. A 5-point smoothing was applied to the error values to simulate the 5-point smoothing used in operational processing. The standard deviation for the error values was found to be 0.015 arcsec and for the smoothed error values 0.0066 arcsec.

These results indicate that while the $0.02 \mathrm{arcsec}$ precision obtained using this approach seems surprising, it is well within predicted performance. It is believed that much of the remaining difference between estimated and observed precision is due to correlated noise along pixel rows, which prevents the ideal noise reduction expected from random error statistics. The shift-variant error due to pixel nonuniformity also produces error for events that incur exceptionally large spacecraft pointing drift. The original SOFIE design included a scan mirror that would have fixed the solar image at high altitudes to \pm 0.5 arcsec of movement on the FPA. Unfortunately, that mirror system was damaged during testing, forcing replacement with a fixed mirror and reliance on spacecraft pointing. Although the spacecraft has performed exceptionally, the drift during an event can be many arcseconds, substantially increasing the shift-variant error. Nevertheless, the analysis demonstrates the precision potential of this edge tracking approach, using the large statistical advantages afforded by modern high-density 2D detector arrays. It also shows that results are theoretically consistent with the Sun sensor performance.

\section{Conclusion}

This paper presents results of a new approach to measuring light bending angles (refraction angles) using solar extent measurements during occultation. A Sun sensor used by the SOFIE instrument on the AIM satellite demonstrates refraction angle measurements with a precision of approximately
0.02 arcsec and systematic error of $<1.0 \%$. The key to achieving this quality is the ability to derive refraction from the solar extent measurements. The approach eliminates error due to pointing uncertainty, both mean and jitter, and renders most systematic errors to fractional scale errors.

Temperature and pressure profiles derived from these measurements show $\pm 2 \mathrm{~K}$ or better accuracy from tropospheric cloud top (or $5 \mathrm{~km}$, whichever is higher) through the stratosphere, with $\pm 5^{\circ}$ accuracy to $60 \mathrm{~km}$ and potentially as high as $70 \mathrm{~km}$ by averaging multiple events. The dominant unquantified systematic error in retrieved temperature is believed due to dynamic conditions, particularly in the winter hemisphere, which can cause significant deviation from the spherical symmetry assumption in the analysis.

This approach provides a method of obtaining coaligned temperature/pressure profiles extending up into the lower mesosphere for occultation sensors using solar imaging with modern inexpensive 2D detector arrays. With higher density and higher $S / N$ arrays, optimized imaging configuration and active pointing control, there appears to be few obstacles to achieving a precision of a few milliarcseconds (tens of nanoradians), which if achieved could provide temperature profiles for single occultation events to $70 \mathrm{~km}$.

The AIM mission is funded by the National Aeronautics and Space Administration (NASA) Small Explorer program. The mission is led and managed by Principal Investigator James Russell of Hampton University. SOFIE was built by the Space Dynamics Laboratory (SDL) at Utah State University. We are indebted to the SDL team for their tireless dedication over many years and through many trials, that resulted in an outstanding instrument. Thanks to Orbital Sciences Corporation for their efforts in providing a great spacecraft, with a high precision pointing system that was critical to SOFIE performance. Thanks to the Laboratory for Atmospheric and Space Physics (LASP) for their technical guidance in making the AIM mission, and SOFIE, a success. And thanks to all the AIM team members for contributions and support during the past years.

\section{References}

1. L. M. Jones, F. F. Fischbach, and J. W. Peterson, "Satellite measurements of atmospheric structure by refraction," Planet. Space Sci. 9, 351-352 (1962).

2. G. M. Grechko, A. S. Gurvich, V. A. Lyakhov, S. A. Savchenko, and S. V. Sokolovskiy, "Results of an investigation of refraction during the third expedition on the Salyut-6 Orbiter," Izv. Russ. Acad. Sci. Atmos. Oceanic Phys., Engl. Transl. 17, 835-841 (1981).

3. R. L. White, W. E. Tanner, and R. S. Polidan, "Star line-of-sight refraction observations from the orbiting astronomical observatory Copernicus and deduction of stratospheric structure in the tropical region,” J. Geophys. Res. 88, 8535-8542 (1983).

4. R. J. Vervack, Jr., J. H. Yee, J. F. Carbary, and F. Morgan, "Atmospheric remote sensing using a combined extinctive and stellar occultation technique. 3. Inversion methods for 
refraction measurements,” J. Geophys. Res. 107, doi:10.1029/ 2001JD000796 (2002).

5. D. M. Ward and B. M. Herman, "Refractive sounding by use of satellite solar occultation measurements including an assessment of its usefulness to the Stratospheric Aerosol and Gas Experiment Program," Appl. Opt. 37, 8306-8317 (1998).

6. E. R. Kursinski, G. A. Hajj, W. I. Bertiger, S. S. Leroy, T. K. Meehan, L. J. Romans, J. T. Schofield, D. J. McCleese, W. G. Melbourne, C. L. Thornton, T. P. Yunck, J. R. Eyre, and R. N. Nagatani, "Initial results of radio occultation observations of Earth's atmosphere using the Global Positioning System," Science 271, 1107-1110 (1996).

7. R. Ware, M. Exner, D. Feng, M. Gorbunov, K. Hardy, B. Herman, Y. Kuo, T. Meehan, W. Melbourne, C. Rocken, W. Schreiner, S. Sokolovsky, F. Solheim, X. Zou, R. Anthes, S. Businger, and K. Trenberth, "GPS sounding of the atmosphere from low Earth orbit: preliminary results," Bull. Am. Meteorol. Soc. 77, 19-40 (1996).

8. K. Hocke, "Inversion of GPS meteorology data," Ann. Geophys. 15, 443-450 (1997).

9. A. K. Steiner, G. Kirchengast, and H. P. Ladreiter, "Inversion, error analysis and validation of GPS/MET occultation data," Ann. Geophys. 17, 122-138 (1999).

10. G. Fjeldbo, A. J. Kliore, and V. R. Eshleman, "The neutral atmosphere of Venus as studied with the Mariner V Radio Occultation Experiments," Astron. J. 76, 123-140 (1971).
11. R. A. Phinney and D. C. Anderson, "On the radio occultation method for studying planetary atmospheres," J. Geophys. Res. 73, 1819-1827 (1968).

12. B. Edlen, "The refractive index of air," Metrologia 2, 71-80 (1966).

13. P. E. Ciddor and R. J. Hill, "Refractive index of air. 2. Group index," Appl. Opt. 38, 1663-1667 (1999).

14. L. L. Gordley, M. Hervig, C. Fish, J. M. Russell III, S. Bailey, J. Cook, S. Hansen, A. Shumway, G. Paxton, L. Deaver, T. Marshall, J. Burton, B. Magill, C. Brown, E. Thompson, and J. Kemp, "The Solar Occultation For Ice Experiment (SOFIE)," J. Atmos. Solar-Terr. Phys. 71, 300-315, doi:10.1016/j.jastp.2008.07.012 (2009).

15. M. A. Whiteley, "Design of a space-borne Sun tracking system.," M.S. thesis, (Utah State University, 2006).

16. J. C. Burton, K. W. Miller, and S. K. Park, "Rectangularly and hexagonally sampled imaging system fidelity analysis," Proc. SPIE 1961, 81-92 (1993).

17. S. E. Reichenbach, S. K. Park, and R. Narayanswamy, "Characterizing digital image acquisition devices,” Opt. Eng. 30, 170-177 (1991).

18. L. L. Gordley, M. J. McHugh, M. E. Hervig, J. C. Burton, L. Liu, B. E. Magill, and J. M. Russell, "Temperature, pressure and high-fidelity pointing knowledge for solar occultation using 2D focal plane arrays," Proc. SPIE 5883, 588310 (2005).

19. A. E. Hedin, "Extension of the MSIS Thermosphere Model into the middle and lower atmosphere," J. Geophys. Res. 96, 1159-1172 (1991). 